\title{
Complete Complementary DNA-derived Amino Acid Sequence of Canine Cardiac Phospholamban
}

\author{
Junichi Fujii, Atsuko Ueno," Katsuhiko Kitano,* Shoji Tanaka,* Masaaki Kadoma, and Michihiko Tada \\ Division of Cardiology, First Department of Medicine, and Department of Pathophysiology, Osaka University School of Medicine, \\ Fukushima-ku, Osaka 553; and *Suntory Institute for Biomedical Research, Shimamoto-cho, Mishima-gun, Osaka 618, Japan
}

\begin{abstract}
Complementary DNA (cDNA) clones specific for phospholamban of sarcoplasmic reticulum membranes have been isolated from a canine cardiac cDNA library. The amino acid sequence deduced from the cDNA sequence indicates that phospholamban consists of 52 amino acid residues and lacks an amino-terminal signal sequence. The protein has an inferred mol wt 6,080 that is in agreement with its apparent monomeric mol wt 6,000 , estimated previously by sodium dodecyl sulfate-polyacrylamide gel electrophoresis. Phospholamban contains two distinct domains, a hydrophilic region at the amino terminus (domain I) and a hydrophobic region at the carboxy terminus (domain II). We propose that domain I is localized at the cytoplasmic surface and offers phosphorylatable sites whereas domain II is anchored into the sarcoplasmic reticulum membrane.
\end{abstract}

\section{Introduction}

Protein phosphorylation catalyzed by cyclic AMP (cAMP)-dependent protein kinase (EC 2.7.1.37, ATP:protein phosphotransferase) has been postulated to play a pivotal role in the regulation of excitation-contraction coupling of myocardium (1). In canine cardiac sarcoplasmic reticulum (SR) ${ }^{1}$, an integral membrane protein termed phospholamban (2) serves as the major substrate for phosphorylation by a cAMP-dependent protein kinase. This protein was also reported to be phosphorylated by $\mathrm{Ca}^{2+}$, calmodulin-dependent protein kinase $(3,4)$, and protein kinase C (5). cAMP-dependent phosphorylation of serine residues in phospholamban (6) is associated with a marked stimulation of $\mathrm{Ca}^{2+}$ pumping function by SR (7-9). A similar regulatory mechanism is considered to mediate $\beta$-adrenergic inotropic effects of catecholamines in myocardial cells (1). Phospholamban was suggested to exhibit a unique molecular assembly in that the holoprotein of $25-27 \mathrm{kD}$ may consist of five monomers (10-12). The oligomeric form was thought to be

Address reprint requests to Michihiko Tada, First Department of Medicine, Osaka University School of Medicine, Fukushima-ku, Osaka 553, Japan.

Received for publication 6 August 1986.

1. Abbreviation used in this paper: SR, sarcoplasmic reticulum.

J. Clin. Invest.

(c) The American Society for Clinical Investigation, Inc.

0021-9738/87/01/0301/04 \$1.00

Volume 79, January 1987, 301-304 stable in sodium dodecyl sulfate above its critical micelle concentration at low temperature, with the probable molecular size of the monomeric species being 5-6 $\mathrm{kD}(12,13)$.

When phospholamban purified by our method (14) was subjected to sequence determination, we identified 35 consecutive amino acid residues starting from an $\mathrm{N}^{\alpha}$-acetylated methionine (13). This enabled us to isolate and sequence the complementary DNA (cDNA) clones encoding canine cardiac phospholamban. This paper describes the molecular cloning and complete amino acid sequence of phospholamban deduced from cDNA analysis.

\section{Methods}

Construction of a cDNA library. Total RNA originating from cardiac ventricular muscle of adult dog was extracted in $4 \mathrm{M}$ guanidine thiocyanate buffer as described by Chirgwin et al. (15). Poly(A) ${ }^{+}$RNA was prepared by twice subjecting the total RNA preparation to oligo(dT)cellulose column chromatography (16). A cDNA library was constructed as described by Okayama and Berg (17) with $14 \mu \mathrm{g}$ of poly(A) ${ }^{+}$RNA and $2 \mu \mathrm{g}$ of vector/primer DNA. Escherichia coli DH1 was used for transformation. $\sim 8,300$ ampicillin-resistant transformants were obtained per microgram of starting messenger RNA (mRNA).

Screening of the cDNA library with oligodeoxyribonucleotides. 32 oligodeoxyribonucleotides composed of all possible complementary sequences predicted for a partial amino acid sequence Glu 19-Met-ProGln-Gln-Ala 24 of phospholamban (13), excluding the third nucleotide residue of the alanine codon, were synthesized by the phosphoramidate method (18). To isolate cDNA clones for phospholamban, the cDNA library was screened by colony hybridization with the $5^{\prime}-{ }^{32}$ P-labeled synthetic oligodeoxyribonucleotide probes described by Wood et al. (19).

\section{Results and Discussion}

Isolation of $c D N A$ clones. A canine cardiac cDNA library was screened by hybridization with a mixture of 32 synthetic oligodeoxyribonucleotide probes as described in Methods. Three hybridization-positive clones were isolated from about 3,000 transformants. Recombinant plasmids from selected clones were digested with various restriction enzymes and electrophoresed in agarose gel to determine the size. All these plasmids contained the same size insert of $\sim 800$ bases with the same restriction maps, suggesting that they were derived from the same mRNA. Among these three plasmids, one plasmid termed as pPLB1 was sequenced according to the strategy indicated in Fig. 1.

Nucleotide sequence of $c D N A$. Fig. 2 depicts the complete nucleotide sequence of the cDNA insert of the pPLB1 plasmid, having 832 base pairs. Analysis of the cloned cDNA showed an open reading frame of 156 nucleotides ( 52 codons) starting with the ATG codon (position 1) and ending with the TGA stop codon (position 157). 

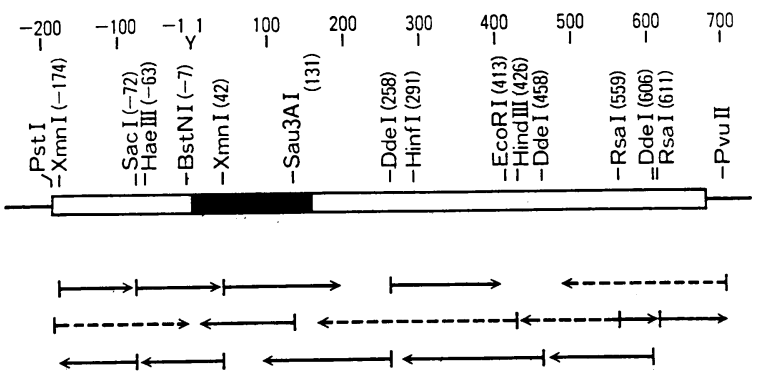

Figure 1. Restriction map and sequencing strategy of pPLB1 insert. The restriction map displays restriction endonuclease sites relevant to the present work. They are represented by numbers indicating the $5^{\prime}-$ terminal nucleotides generated by cleavage. Nucleotides are numbered as in Fig. 2. Solid box represents the cording region. Open boxes represent the 5'- and 3'-untranslated regions. Solid lines shown below the restriction map indicate the nucleotide sequence determined by the dideoxy chain termination method of Sanger et al. (20) after the fragments of the insert generated by digestion with respective endonucleases were subcloned in M13mp18 or M13mp19 (21). Dashed lines indicate the nucleotide sequence determined by the Maxam and Gilbert method (22). The direction and extent of sequence determinations are shown by arrows.

Fig. 2 also shows the amino acid sequence deduced from the 52 codons. In this reading frame, the sequence of nucleotide residues $1-105$, which encode amino acids 1-35, corresponded precisely to the 35 amino-terminal sequence from Acetyl-Met 1 to Phe 35 (13). The two residues unidentified by Edman degradation for purified phospholamban, positions 36 and 41 (13), were both deduced to be cysteine residues. Seven amino acids following Ile 45 would represent carboxy-terminal residues ending at Leu 52, although protein sequencing is necessary to confirm these findings.

The cloned cDNA extended for 180 nucleotides to the $5^{\prime}$ end of the sequence coding for the amino terminus. Because this sequence contained an in-frame stop codon (TGA, position -78) and not an ATG codon between the TGA and the Met 1, we conclude that phospholamban is synthesized without an amino-terminal signal sequence. Therefore, it is likely that the information required for integration of phospholamban into the membrane is encoded in an uncleavable signal sequence in the carboxy terminus.

The observed nucleotide context of the initiator ATG codon (GXXATGG), which has also been found in other eukaryotic mRNAs (23), is thought to diverge from the most commonly found consensus sequence, AXXATGG (24). The 3 '-untranslated region terminated in a poly(A) tail (about 30 consecutive $A$ residues). The sequence upstream of the tail did not contain a perfect AATAAA, the so-called polyadenylation signal (25), but the similar sequence AATGAA was located at 24 bases before the poly(A) tail.

Molecular characteristics of phospholamban. The molecular weight of encoded protein was estimated to be 6,080 , in good agreement with the apparent molecular weight of phospholamban monomer $(6,000) \cdot(12,13)$. The hydropathic profile $(26)$ of phospholamban (Fig. 3) indicates that the protein is amphipathic in nature. The protein is divided into two domains. The aminoterminal domain from Met 1 to Asn 30 (domain I) was composed largely of hydrophilic amino acids, whereas the other domain representing 22 amino acids from Leu 31 to Leu 52 (domain II) was highly hydrophobic. Domain I, but not domain II, contained phosphorylatable serine and threonine residues, indicating that domain I faces cytoplasmic surface of SR membrane. Quite recently, Simmerman et al. (27) reported a partial amino acid sequence and phosphorylation sites in phospholamban. They indicated 36 amino acid residues corresponding precisely to those in the peptide from Ser 10 to Ile 45 in Fig. 2. Phosphorylation sites at Ser 7 and Thr 8 in their sequence would correspond to Ser 16 and Thr 17 in our sequence, respectively.

Secondary structure prediction of the protein $(28,29)$ suggested that domain I consists of an $\alpha$-helix (Fig. 3). In view of an unusual behavior of phospholamban molecule $(10,11)$, it is intriguing to ask whether this helix breaks into two portions, possibly at around Pro 21 , thus allowing side chains of each to express hydrophobic interactions (29). Although alternative molecular models are possible, such an assumption may explain phosphorylation-induced structural changes of phospholamban molecule that lead to profound functional consequences. Thus phosphorylation of Ser 16 and/or Thr 17 might induce an al-

$$
5 \text { '-AGAAAACTTTCTAACTAAACAC -159 }
$$

CGATAAGACTTCATACAACTCACAATACTTTATATTGTAATCATCACAAGAGCCAAGGCTACCTAAAAGAAGAGAGTGG $\quad-80$ TTGAGCTCACATTTGGCCGCCAGCTTTTTACCTTTCTCTTCACCATTTAAAACTTGAGACTTCCTGCTTTCCTGGGTC -1

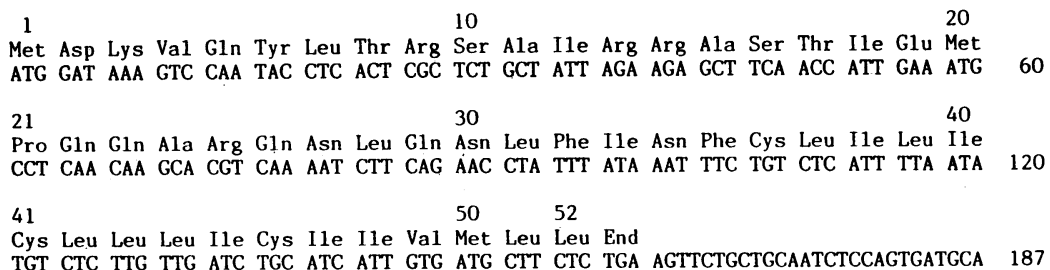
TGT CTC TTG TTG ATC TGC ATC ATT GTG ATG CTT CTC TGA AGTTCTGCTGCAATCTCCAGTGATGCA 187 ACTTGTCACCATCAACTTAATATCTGCCATCCCATGAAGAGGGGAAAATAATACTATATAACAGACCACTTCTAAGTAG 266 AAGATTTTACTTGTGAAAAGGTCAAGATTCAGAACAAAAGAAATTATTAACAAATGTCTTCATCTGTGGGATTTTGTAA 345 ACATGAAAAGAGCTTTATTTTCAAAAATTAACTTCAAAATGACTATAGGTGCGCATAATGTAATTGCTGAATTCCTCAA 424 CAAAGCTTGTAAAAGTTTCTATGCCAAATTTTTTCTGAGGGTAAAGTAGGAGTTTAGTTTTAAAACTGCTCTGCTAACC 503 AGTTCACTTCACATATAAAGCATTAGCTTCACTATTTGAGCTAAATATTTATATTGTACTGTAAATGCCTATGTAATGT 582 TTATTAAGATTTTTCAAGTCTCCGC'TAAGTACGAAATAATCATCCAAATGAAGTCATCATTTGAAATAGC- ${ }^{\prime}$ 652
Figure 2. Nucleotide sequence of pPLBl cDNA and deduced amino acid sequence of canine cardiac phospholamban. Nucleotide residues are numbered in the $5^{\prime}$-to-3' direction, beginning with the first residue of ATG triplet encoding the initiator methionine. $\mathrm{Nu}$ cleotides on the $5^{\prime}$ side of residue 1 are indicated by negative numbers. A poly(A) tail on $3^{\prime}$ end is not shown. The predicted amino acid sequence of phospholamban is displayed above the nucleotide sequence. Its residue number begins with the initiator methionine. Box indicates the presumptive polyadenylation signal. The in-frame stop codon preceding the initiator codon is underlined. 


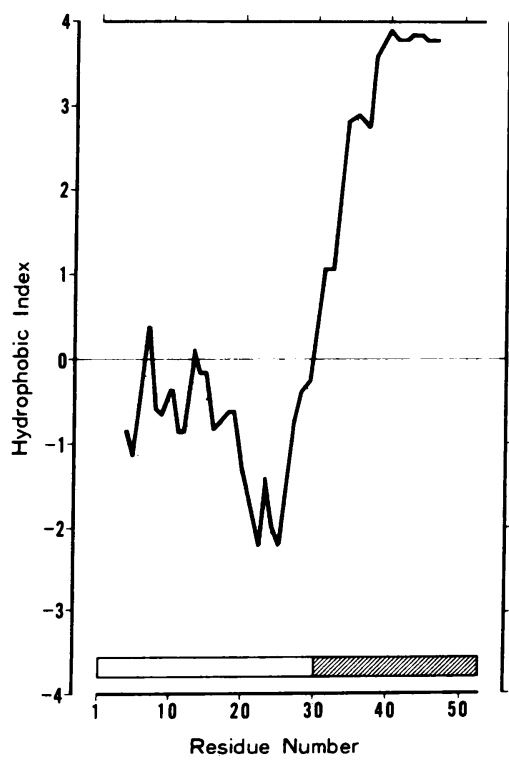

Figure 3. Hydropathy profile and predicted secondary structure of phospholamban. The ordinate represents the average hydropathy across a 9-amino-acid window centered at a given residue (26). The position of the predicted $\alpha$-helix $(28,29)$ is shown by an open box, putative transmembrane helix position is shown by a cross-hatched box.

teration in charge distribution of the helical unit and would result in a conformational change of domain I. This hypothesis would presumably account for an electrophoretic mobility shift induced by phosphorylation $(10,11)$. It remains to be examined whether this assumption could also be interpreted as phosphorylation-induced actions of phospholamban on the Ca pump ATPase (1). Domain II, consisting largely of hydrophobic amino acids, would also form an $\alpha$-helix that allows a hydrophobic interaction with membrane lipids. Three cysteine residues are reported to exist as free sulfhydryl groups (12). Domain II consisting of 22 amino acids is clearly immersed in the lipid bilayer of SR membrane and could anchor phospholamban in the membrane.

It is interesting to speculate about which portion of phospholamban protomer would permit intermolecular interaction to form the pentameric assembly. A tryptic fragment of phospholamban holoprotein that was devoid of the phosphorylation site remained pentameric (30). Since trypsinization under these conditions results in the removal of amino-terminal residues up to $\operatorname{Arg} 25$, the ability to maintain an oligomeric organization would reside in the carboxy-terminal residues, possibly in the carboxyl end of domain I or in domain II. Phospholamban was previously proposed to exert control over the Ca pump ATPase of cardiac SR (1). However, no structural evidence has yet been presented to support such a molecular interaction with a $\mathrm{Ca}$ pump ATPase. Suzuki and Wang (31) observed that a monoclonal antibody directed against phospholamban blocked the phosphorylation and dephosphorylation of phospholamban but markedly increased the ATP-dependent $\mathrm{Ca}^{2+}$ pump activity by cardiac SR. Possibly the residues near phosphorylation sites in domain I are responsible for exerting an influence over the $\mathrm{Ca}$ pump ATPase. It remains to be examined which domain of the Ca pump ATPase interacts with phospholamban.

\section{Acknowledgments}

We are greatly indebted to Professor Fumio Sakiyama and Dr. Hiroko Toda, Institute for Protein Research, Osaka University, for their cooperation in determining the partial amino acid sequence of phospholamban. We also thank Dr. Teruhisa Noguchi, Suntory Institute for Biomedical Research, for his continuous encouragement during the course of this study.
This work was supported by research grants from the Ministries of Education, Science and Culture, and Health and Welfare of Japan and a Grant-in-Aid from the Muscular Dystrophy Association of America.

\section{References}

1. Tada, M., and A. M. Katz. 1982. Phosphorylation of the sarcoplasmic reticulum and sarcolemma. Annu. Rev. Physiol. 44:401-423.

2. Tada, M., M. A. Kirchberger, and A. M. Katz. 1975. Phosphorylation of a 22,000-dalton component of the cardiac sarcoplasmic reticulum by adenosine $3^{\prime}: 5^{\prime}$-monophosphate-dependent protein kinase. $J$. Biol. Chem. 250:2640-2647.

3. Le Peuch, C. J., J. Haiech, and J. G. Demaille. 1979. Concerted regulation of cardiac sarcoplasmic reticulum calcium transport by cyclic adenosine monophosphate dependent and calcium-calmodulin-dependent phosphorylations. Biochemistry. 18:5150-5157.

4. Tada, M., M. Inui, M. Yamada, M. Kadoma, T. Kuzuya, H. Abe, and S. Kakiuchi. 1983. Effects of phospholamban phosphorylation catalyzed by adenosine $3^{\prime}: 5^{\prime}$-monophosphate- and calmodulin-dependent protein kinases on calcium transport ATPase of cardiac sarcoplasmic reticulum. J. Mol. Cell. Cardiol. 15:335-346.

5. Movsesian, M. A., M. Nishikawa, and R. S. Adelstein. 1984. Phosphorylation of phospholamban by calcium-activated, phospholipid-dependent protein kinase. J. Biol. Chem. 259:8029-8032.

6. Kirchberger, M. A., M. Tada, and A. M. Katz. 1974. Adenosine $3^{\prime}: 5^{\prime}$-monophosphate-dependent protein kinase-catalyzed phosphorylation reaction and its relationship to calcium transport in cardiac sarcoplasmic reticulum. J. Biol. Chem. 249:6166-6173.

7. Tada, M., M. A. Kirchberger, D. I. Repke, and A. M. Katz. 1974. The stimulation of calcium transport in cardiac sarcoplasmic reticulum by adenosine $3^{\prime}: 5^{\prime}$-monophosphate-dependent protein kinase. J. Biol. Chem. 249:6174-6180.

8. Tada, M., F. Ohmori, M. Yamada, and H. Abe. 1979. Mechanism of the stimulation of $\mathrm{Ca}^{2+}$-dependent ATPase of cardiac sarcoplasmic reticulum by adenosine $3^{\prime}: 5^{\prime}$-monophosphate-dependent protein kinase. J. Biol. Chem. 254:319-326.

9. Tada, M., M. Yamada, F. Ohmori, T. Kuzuya, M. Inui, and H. Abe. 1980. Transient state kinetic studies of $\mathrm{Ca}^{2+}$-dependent ATPase and calcium transport by cardiac sarcoplasmic reticulum. J. Biol. Chem. 255:1985-1992.

10. Imagawa, T., T. Watanabe, and T. Nakamura. 1986. Subunit structure and multiple phosphorylation sites of phospholamban. $J$. Biochem. (Tokyo). 99:41-53.

11. Wegener, A. D., and L. R. Jones. 1984. Phosphorylation-induced mobility shift in phospholamban in sodium dodecyl sulfate-polyacrylamide gels. J. Biol. Chem. 259:1834-1841.

12. Jones, L. R., H. K. B. Simmerman, W. W. Wilson, F. R. N. Gurd, and A. D. Wegener. 1985. Purification and characterization of phospholamban from canine cardiac sarcoplasmic reticulum. J. Biol. Chem. 260:7721-7730.

13. Fujii, J., M. Kadoma, M. Tada, H. Toda, and F. Sakiyama. 1986. Characterization of structural unit of phospholamban by amino acid sequencing and electrophoretic analysis. Biochem. Biophys. Res. Commun. 138:1044-1050.

14. Inui, M., M. Kadoma, and M. Tada. 1985. Purification and characterization of phospholamban from canine cardiac sarcoplasmic reticulum. J. Biol. Chem. 260:3708-3715.

15. Chirgwin, J. M., A. E. Przybyla, R. J. MacDonald, and W. J. Rutter. 1979. Isolation of biologically active ribonucleic acid from sources enriched in ribonuclease. Biochemistry. 18:5294-5299.

16. Nakazato, H., and M. Edmonds. 1974. Purification of messenger RNA and heterogeneous nuclear RNA containing poly(A) sequences. Methods Enzymol. 29:431-443.

17. Okayama, H., and P. Berg. 1982. High-efficiency cloning of fulllength cDNA. Mol. Cell. Biol. 2:161-170.

18. Beaucage, S. L., and M. H. Caruthers. 1981. Deoxynucleoside phosphoramidites-a new class of key intermediates for deoxypolynucleotide synthesis. Tetrahedron Lett. 22:1859-1862. 
19. Wood, W. I., J. Gitschier, L. A. Lasky, and R. M. Lawn. 1985. Base composition-independent hybridization in tetramethylammonium chloride: a method for oligonucleotide screening of highly complex gene libraries. Proc. Natl. Acad. Sci. USA. 82:1585-1588.

20. Sanger, F., S. Nicklen, and A. R. Coulson. 1977. DNA sequencing with chain-terminating inhibitors. Proc. Natl. Acad. Sci. USA. 74:54635467.

21. Yanisch-Perron, C., J. Vieira, and J. Messing. 1985. Improved M13 phage cloning vectors and host strains: nucleotide sequences of the M13mp18 and pUC19 vectors. Gene (Amst.). 33:103-119.

22. Maxam, A. M., and W. Gilbert. 1980. Sequencing end-labeled DNA with base-specific chemical cleavages. Methods Enzymol. 65:499560 .

23. MacLennan, D. H., C. J. Brandl, B. Korczak, and N. M. Green 1985. Amino-acid sequence of a $\mathrm{Ca}^{2+}+\mathrm{Mg}^{2+}$-dependent ATPase from rabbit muscle sarcoplasmic reticulum, deduced from its complementary DNA sequence. Nature (Lond.). 316:696-700.

24. Kozak, M. 1984. Point mutations close to the AUG initiator codon affect the efficiency of translation of rat preproinsulin in vivo. Nature (Lond.). 308:241-246.
25. Proudfoot, N. J., and G. G. Brownlee. 1976. 3Non-coding region sequences in eukaryotic messenger RNA. Nature (Lond.). 263:211-214.

26. Kyte, J., and R. F. Doolittle. 1982. A simple method for displaying the hydropathic character of a protein. J. Mol. Biol. 157:105-132.

27. Simmerman, H. K. B., J. H. Collins, J. L. Theibert, A. D. Wegener, and L. R. Jones. 1986. Sequence analysis of phospholamban: identifcation of phosphorylation sites and two major structural domains. $J$. Biol. Chem. 261:13333-13341.

28. Chou, P. Y., and G. D. Fasman. 1978. Empirical predictions of protein conformation. Annu. Rev. Biochem. 47:251-276.

29. Schiffer, M., and A. B. Edmundson. 1967. Use of helical wheels to represent the structures of proteins and to identify segments with helical potential. Biophys. J. 7:121-135.

30. Wegener, A. D., H. K. B. Simmerman, J. Liepnieks, and L. R. Jones. 1986. Proteolytic cleavage of phospholamban purified from canine cardiac sarcoplasmic reticulum vesicles. J. Biol. Chem. 261:5154-5159.

31. Suzuki, T., and J. H. Wang. 1986. Stimulation of bovine cardiac sarcoplasmic reticulum $\mathrm{Ca}^{2+}$ pump and blocking of phospholamban phosphorylation and dephosphorylation by a phospholamban monoclonal antibody. J. Biol. Chem. 261:7018-7023. 